\title{
Classification of Stabilometric Time-Series Using an Adaptive Fuzzy Inference Neural Network System
}

\author{
Juan A. Lara ${ }^{1}$, Pari Jahankhani ${ }^{2}$, Aurora Pérez ${ }^{1}$, Juan P. Valente ${ }^{1}$, \\ and Vassilis Kodogiannis ${ }^{2}$ \\ ${ }^{1}$ Technical University of Madrid, School of Computer Science, Campus de Montegancedo, \\ 28660, Boadilla del Monte, Madrid, Spain \\ j.lara.torralbo@upm.es, \{aurora, jpvalente\}@fi.upm.es \\ ${ }^{2}$ University of Westminster, School of Electronic and Computer Science, \\ London HA1 3TP, United Kingdom \\ \{parij, kodogiv\}@wmin.ac.uk
}

\begin{abstract}
Stabilometry is a branch of medicine that studies balance-related human functions. The analysis of stabilometric-generated time series can be very useful to the diagnosis and treatment balance-related dysfunctions such as dizziness. In stabilometry, the key nuggets of information in a time series signal are concentrated within definite time periods known as events. In this study, a feature extraction scheme has been developed to identify and characterise the events. The proposed scheme utilises a statistical method that goes through the whole time series from the start to the end, looking for the conditions that define events, according to the experts' criteria. Based on these extracted features, an Adaptive Fuzzy Inference Neural Network has been applied for the classification of stabilometric signals. The experimental results validated the proposed methodology.
\end{abstract}

\section{Introduction}

Stabilometry is the branch of medicine responsible for examining balance in human beings. Balance and dizziness disorders are probably two of the most common illnesses that physicians have to deal with. Around $30 \%$ of population suffers from any kind of dizziness disorder before reaching the age of 65; for older people, this pathologic symptom occurs in a more frequent rate, and it is responsible for people's0020falling. In order to examine balance, a device, called posturograph, has been used to measure the balance-related functionalities. The patient stands on a platform and completes a series of tests, as shown in Fig. 1. These tests have been designed to isolate the main sensorial, motor and biomechanical components that contribute to balance. Emphasis has been given to the evaluation of the capacity for each individual components as well as the overall components capacity. The posturograph generates a time-series signal, where the main information normally is confined to specific regions of the series, known as events [1]. Similarities exist also at many other domains. In seismography, for example, the regions of interest are when the time series indicates an earthquake, volcanic activity leading up to the quake or replications. 
Initially, stabilometry was considered as a technique measuring only the balance of human beings under certain conditions [2] [3]. Many researchers have studied the effect of closed eyes on balance. [4] and [5]. These works confirmed that the condition of having the eyes closed affects balance due to the fact that balance has a strong visual component.

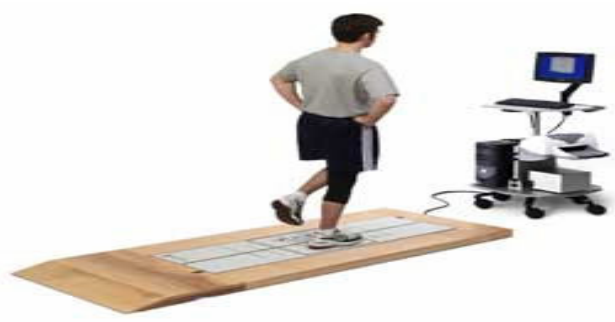

Fig. 1. Patient completing a test on a posturograph

Currently, stabilometry is also considered as a useful tool for diagnosing balancerelated disorders like the Parkinson disease [6] or benign vertigo of childhood [7]. Regarding stabilometric data analysis, body sway parameters have been used for analysis balance-related functions [8], [9]. However, it appears that classic posturographic parameters, such as the measure of the sway of the centre of pressure [10] have failed in the detection of balance disorders [11]. The analysis of stabilometric time series using data mining techniques offers new possibilities. Recently, a new method has been developed for comparison of two stabilometric time-series [12]. This method calculates the level of similarity of two time-series and can be applied to compare either the balance of two patients or to study how the balance of one patient evolves with time. Stabilometry also plays an important role in the treatment of balance-related diseases. The NedSVE/IBV system [3] has been utilised for the development of a new method that assists in the rehabilitation of patients who have lost their balance [13].

With the continuously growing demand for models for complex systems inherently associated with nonlinearity, high-order dynamics, time-varying behaviour, and imprecise measurements, such as stabilometric time-series, there is a need for a relevant modelling environment. Efficient modelling techniques should utilise features/pertinent variables extracted from raw data and "transform" them is a highly representative dataset. The models should also be able to take advantage of the existing domain knowledge (such as a prior experience of human observers or operators) and augment it by available numeric data to form a coherent data knowledge modelling entity.

Fuzzy systems accept numeric inputs and convert theses into linguistic values (represented by fuzzy numbers) that can be manipulated with linguistic IF-THEN rules and with fuzzy logic operations, such as fuzzy implication and composition rules of inference. However, at present there is no systematic procedure for the design of a fuzzy system. Usually the fuzzy rules are generated by converting human operators' experience into fuzzy linguistic form directly and by summarizing the system behaviour (sampled input-output pairs) of the operators. During the last years, the 
fuzzy neural network approach has gained considerable interest for solving real world problems, including modelling and control of highly complex systems, signal processing and pattern recognition [14].

In this paper, we will consider an Adaptive Fuzzy Inference Neural Network system (AFINN) for the classification of features extracted from stabilometric events. AFINN is made up of Gaussian-membership functions associated with local linear systems. The proposed fuzzy logic system is based on the Sugeno type modified with the introduction of an additional layer of output partitions and constructs its initial rules by clustering while the final fuzzy rule base is determined by competitive learning [15].

\section{Data Recording}

Throughout this research, a static Balance Master posturograph has been used. In a static posturograph, the platform on which the patient stands is static, i.e. does not move. The platform has four sensors, one at each of the four corners: right-front (RF), left-front (LF), right-rear (RR) and left-rear (LR). Each sensor records a datum every 10 milliseconds during the test. This datum is sent to the computer connected to the posturograph. The datum is the intensity of the pressure that the patient is exerting on that sensor. Data are recorded as multidimensional time-series.

The posturograph Balance Master can be used to run a wide range of tests according to a predefined protocol. This research study has focused on the Unilateral Stance (UNI) test that is the most useful for domain experts (physicians) in terms of output information. UNI test aims to measure how well the patient is able to keep his or her balance when standing on one leg with either both eyes open or both eyes closed for 10 seconds. The UNI test generates time-series signals containing events, that is, regions of special interest for experts in the domain. Next section describes the possible events appearing in the time series of UNI test and the features used to characterise these events. Both the events and their features were determined according to the physicians' criteria. The following cases are the four different conditions of UNI test:

- Left leg with Open Eyes: The patient is asked to hold still with his or her left leg on the platform while his or her right leg has to be lifted.

- Right leg with Open Eyes: The patient is asked to hold still with his or her right leg on the platform while his or her left leg has to be lifted.

- Left leg with Closed Eyes: The patient is asked to hold still with his or her left leg on the platform while his or her right leg has to be lifted.

- $\quad$ Right leg with Closed Eyes: The patient is asked to hold still with his or her right leg on the platform while his or her left leg has to be lifted.

Each condition is examined three times (trials) according to the medical protocol. The current research has been carried out on time series from a set of healthy volunteer people aged in the range of 14 to 38 , including both genders and sport and non-sport people. 


\section{Method for the Identification and Feature Extraction of Events}

The physicians are interested in studying the sway velocity (degrees/second) of the patient during the test. The relative absence of sway means stability while greater sway indicates less stability. The sway velocity throughout a whole test is computed as the average of the angular velocity of the patient between each pair of timestamps, according to Eq. 1. Since the UNI test lasts 10 seconds and the sensors measures a datum every 0.01 seconds, the number of timestamps is 1000 .

$$
\text { SwayVelocity }=\frac{\sum_{i=1}^{1000} \text { AngularVelocity }_{i}}{1000}
$$

To compute the angular velocity it is enough to divide the angle $\theta_{i}$ that the patient has moved between the timestamp $i$ and the timestamp $i+1$ by the time between two consecutive timestamps, that is, 0.01 seconds, as shown in Eq. 2.

$$
\text { AngularVelocity }{ }_{i}=\frac{\theta_{i}}{0.01}
$$

In addition to the sway velocity, physicians are interested in the number of events that occur and their more relevant features. There are two types of events in UNI test. The first one occurs when the patient loses balance and puts the lifted leg down onto the platform. This type of event is known in the domain as a fall. When there is a fall, the respective sensors for the lifted leg will register the pressure increase. Fig. 2 shows the time-series of a patient who has taken the UNI test. The curves at the top of the figure are the values recorded by the RR and RF sensors, that is, the right-leg sensors, the leg the patient was standing on. The curves at the bottom of the figure are the values recorded by sensors LR and LF, that is, the left-leg sensors, the leg that should be lifted. The pressures peaks generated when there is a fall event are highlighted. The features characterising the falls are as follows:

- Duration: It is the amount of time between the moment when the patient starts to lose balance and the moment when he or she is stable again, after falling.

- Intensity: It is the strength that the patient exerts on the platform when he or she falls down onto it.

The second type of event occurs when the patient losses balance but, before falling, he or she manages to recover upright position. This kind of event is known in the stabilometric domain as an imbalance. To identify and characterise these kinds of events the method proposed in [12] has been used. It is a statistical method that goes through the time series trying to find points where there is an event.

Regarding fall events, this method calculates the average value of the time-series related to the leg that must be lifted (bottom of Fig. 2). This average value represents the balance value as shown in Fig. 3. The method identifies points where there is a local maximum whose distance to the balance value is higher than a certain threshold $(\partial)$. That distance is precisely the intensity of the fall. The duration of the fall is then calculated by analysing the two intersections between the time series and the balance value line. 


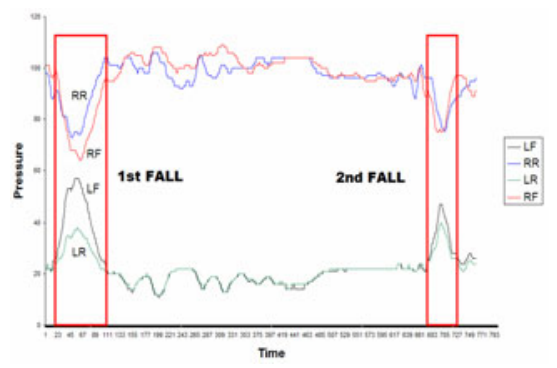

Fig. 2. UNI test time series, highlighting two events (falls)

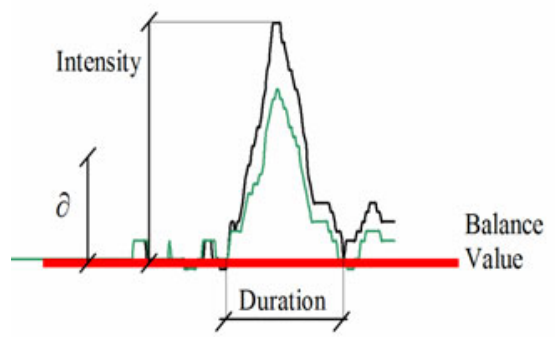

Fig. 3. Fall event taken from a stabilometric time series

In order to classify stabilometric time series, a set of balance-related features have been extracted according to the experts' criteria, as shown in Table 1. These features are as follow:

- Sway Velocity.

- Number of Imbalances.

- Number of Falls.

- Total Duration: It is the sum of the durations of all the falls contained in a time series.

- Maximum intensity: It is the maximum value of intensity of the falls contained in a time series.

These features have been chosen due to the fact that they represent how stable the patient is in a faithful way. The sway velocity is a good overall parameter to study patients' balance. On the other hand, the number of imbalances, the number of falls and the total duration of falls give a clear idea of the amount of time that the patient was unstable during the test.

Table 1. Sample of extracted features

\begin{tabular}{cccccc}
\hline $\begin{array}{c}\text { Time } \\
\text { Series Velocity }\end{array}$ & $\begin{array}{c}\text { Sway } \\
\text { Vumber }\end{array}$ & $\begin{array}{c}\text { Number of } \\
\text { of falls }\end{array}$ & $\begin{array}{c}\text { Total } \\
\text { imbalances }\end{array}$ & $\begin{array}{c}\text { Maximun } \\
\text { Duration }\end{array}$ & \begin{tabular}{c} 
Intensity \\
\hline 1
\end{tabular} \\
\hline 1.92 & 3 & 2 & 31 & 15 \\
2 & 1.18 & 0 & 2 & 0 & 0 \\
$\ldots$ & $\ldots$ & $\ldots$ & $\ldots$ & $\ldots$ & $\ldots$ \\
56 & 1.85 & 0 & 1 & 0 & 0 \\
\hline
\end{tabular}

\section{AFINN Architecture}

In this paper, we will consider an Adaptive Fuzzy Inference Neural Network system (AFINN) which is made up of Gaussian-membership functions associated with local linear systems. The proposed fuzzy logic system is based on the Sugeno type modified with the introduction of an additional layer of output partitions. Unlike the 
ANFIS system, in which the number of local linear systems is same as that of the number of rules, AFINN provides a means of controlling the growth of the number of local linear systems when the order of the system under consideration increases, so that least-squares estimation can be applied without performance degradation. A clustering algorithm is applied for the sample data in order to organise feature vectors into clusters such that points within a cluster are closer to each other than vectors belonging to different clusters. Then fuzzy rule base is created using results obtained from this algorithm. The fuzzy implication of the fuzzy system is based on fuzzy partitions of the input space directly rather than fuzzy partitions of each dimension of the input space. Thus the membership functions considered in the proposed system are multidimensional membership functions. In this sense, there is a similarity with the construction of Gaussian centres in Radial Basis Function networks (RBFN). Since the input space is considered to be partitioned instead of each dimension of the input space, the number of rules can be small and hence the number of local linear systems is also small. In addition, competitive learning technique is applied to locate space partitions according to the clustering of the fuzzy rules at the beginning of training.

The architecture of the proposed neuro-fuzzy network shown in Fig 4 consists of six layers. The first two layers $\mathrm{L}_{1}$ and $\mathrm{L}_{2}$ correspond to IF part of fuzzy rules whereas layers $\mathrm{L}_{4}$ and $\mathrm{L}_{5}$ contain information about THEN part of these rules and perform the defuzzification task.

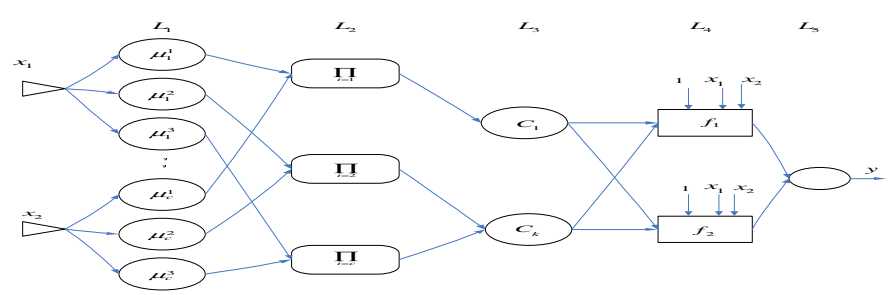

Fig. 4. Structure of AFINN system

The most important problem in fuzzy systems is to find fuzzy rules. Some methods can generate fuzzy rules from input-output pairs [16]. In this paper, the fuzzy rule base is derived using results obtained from a clustering algorithm. The clustering algorithm we apply in this paper at layer $\mathrm{L}_{2}$ consists of two stages. In the first stage the method similar to Learning Vector Quantisation (LVQ) algorithm generates crisp c-partitions of the data set. The number of clusters $c$ and the cluster centres $v_{i}, i=1, \ldots, c$, obtained from this stage are used by FCM (Fuzzy $c$-means) algorithm in the second stage. In layer $\mathrm{L}_{3}$ a mapping between the rule layer and the output layer is performed by a competitive learning process. The local linear systems at $\mathrm{L}_{4}$ are associated with each term of layer $L_{3}$ rather than that of rule base layer $L_{2}$. Thus the size of required matrices for least-squares estimation is considered to be much smaller [15].

\section{Discussion of Results}

Automated diagnostic systems aim to enhance the ability to detect pathological structures in medical examinations and to support evaluation of pathological findings during 
the diagnostic procedure. Most techniques developed for automated stabilometric data analysis have focused on the study of the centre of pressure of the patient. However, balance-related events (falls and imbalances) contain useful information for the physicians. In this research, the proposed AFINN network has been implemented for stabilometric time-series classification, employing the most significant features of the events contained in UNI time series. As explained in section 2, the UNI test consists of four different conditions. This case study focused on the second trial of Left leg with Closed Eyes condition. The first and third trials have not been considered because the first trial contains noise and during the third trial the patient has already learnt how to be stable. In this study, 56 stabilometric time series with 1000 timestamps have been used. The data set was divided into two classes according to the gender of patients: MALE and FEMALE. 38 out of the 56 time series belong to male patients while 18 belong to female patients. 5 balance-related features were extracted from each time series.

In our experiments, the training data set was used to train the AFINN model, whereas the testing data set was used to verify the accuracy and effectiveness of the trained AFINN model for classification of the 2 classes of stabilometric time series. The proposed scheme has high classification accuracy with within 5 epochs. The results of the proposed classifier, using 10 different training sets are illustrated at Table 2.

Table 2. AFINN performance for stabilometric time series

\begin{tabular}{|c|c|c|c|c|}
\hline System & $\begin{array}{c}\text { Rules or } \\
\text { Nodes }\end{array}$ & Epoch & $\begin{array}{c}\text { Class 1 } \\
\text { (Female) }\end{array}$ & $\begin{array}{c}\text { Class 2 } \\
\text { (Male) }\end{array}$ \\
\hline AFFIN & $7 / 4$ & 5 & $95 \%$ & $94.3 \%$ \\
\hline
\end{tabular}

The clustering fuzzification part resulted in 7 rules, while after the competitive layer, the rules were reduced to 4 , which resulted in fewer consequent parameters at the defuzzification layer.

\section{Conclusions}

Fuzzy set theory plays an important role in dealing with uncertainty when making decisions in medical applications. The usage of fuzzy logic enabled us to use the uncertainty in the classifier design and consequently to increase the credibility of the system output. This research study presented a neural network implementation of the AFFIN and its application on the classification of stabilometric time series. The proposed network was trained and tested with the extracted features using a statistical ad-doc method for the identification and the characterisation of events in stabilometric time series. The simulation results reveal an almost perfect performance.

\section{References}

1. Povinelli, R.: Time Series Data Mining: identifying temporal patterns for characterization and prediction of time series, $\mathrm{PhD}$ Thesis, Milwaukee (1999)

2. Romberg, M.H.: Manual of the Nervous Disease of Man, pp. 395-401, Syndenham Society, London (1853) 
3. Baron, J.B., Bobot, J., Bessineton, J.C.: Statokinesimetrie. Presse Med. 64, 36: 863 (1956)

4. Paulus, W.M., Straube, A., Brandt, T.: Visual stabilization of posture: physiological stimulus characteristics and clinical aspects. Brain 107, 1143-1163 (1984)

5. Gagey, P., Gentaz, R., Guillamon, J., Bizzo, G., Bodot-Braeard, C., Debruille, B.C.: Normes 85, Association Française de Posturologie, Paris (1988)

6. Ronda, J.M., Galvañ, B., Monerris, E., Ballester, F.: Asociación entre Síntomas Clínicos y Resultados de la Posturografía Computerizada Dinámica. Acta Otorrinolaringol Esp. 53, 252-255 (2002)

7. Barona, R.: Interés clínico del sistema NedSVE/IBV en el diagnóstico y valoración de las alteraciones del equilibrio. Biomechanics Magazine of the Institute of Biomechanics of Valencia (IBV) (February 2003)

8. Rocchi, L., Chiari, L., Cappello, A.: Feature selection of stabilometric parameters based on principal component analysis. In: Medical \& Biological Engineering \& Computing 2004, vol. 42 (2004)

9. Demura, S., Kitabayashi, T.: Power spectrum characteristics of body sway time series and velocity time series of the center of foot pressure during a static upright posture in preschool children. Sport Sciences for Health 3(1), 27-32 (2008)

10. Diener, H.C., Dichgans, J., Bacher, M., Gompf, B.: Quantification of postural sway in normals and patients with cerebellar diseases. Electroenc. and Clin. Neurophysiol. 57, 134-142 (1984)

11. Corradini, M.L., Fioretti, S., Leo, T., Piperno, R.: Early Recognition of Postural Disorders in Multiple Sclerosis Through Movement Analysis: A Modeling Study. IEEE Transactions on Biomedical Engineering 44(11) (November 1997)

12. Lara, J.A., Moreno, G., Perez, A., Valente, J.P., Lopez-Illescas, A.: Comparing Posturographic Time Series through Event Detection. In: 21st IEEE International Symposium on Computer-Based Medical Systems, CBMS, pp. 293-295 (2008)

13. Peydro, M.F., Vivas, M.J., Garrido, J.D., Barona, R.: Procedimiento de rehabilitación del control postural mediante el sistema NedSVE/IBV. Biomechanics Magazine of the Institute of Biomechanics of Valencia (IBV), Ed. (January 2006)

14. Jang, J.-S.R.: ANFIS: Adaptive-Network-based Fuzzy Inference Systems. IEEE Trans. on Systems, Man and Cybernetics 23, 665-685 (1993)

15. Jahankhani, P., Revett, K., Kodogiannis, V., Lygrouras, J.: Classification Using Adaptive Fuzzy Inference Neural Network. In: Proceedings of the Twelfth IASTED International Conference Artificial Intelligence and Soft Computing (ASC 2008), Palma de Mallorca, Spain, September 1-3 (2008)

16. Sonbol, A., Fadali, M.S.: A new approach for designing TSK fuzzy systems from inputoutput data. In: Proc. of the American Control Conference, vol. 2, pp. 989-994 (2002) 